\title{
Distinct triangle areas in a planar point set over finite fields
}

\author{
Le Anh Vinh \\ University of Education \\ Vietnam National University \\ Hanoi, Vietnam \\ vinhla@vnu.edu.vn
}

Submitted: May 24, 2011; Accepted: Oct 18, 2011; Published: Oct 31, 2011

Mathematics Subject Classification: 05C35, 05C38

\begin{abstract}
Let $\mathcal{P}$ be a set of $n$ points in the finite plane $\mathbb{F}_{q}^{2}$ over the finite field $\mathbb{F}_{q}$ of $q$ elements, where $q$ is an odd prime power. For any $s \in \mathbb{F}_{q}$, denote by $A(\mathcal{P} ; s)$ the number of ordered triangles whose vertices in $\mathcal{P}$ having area $s$. We show that if the cardinality of $\mathcal{P}$ is large enough then $A(\mathcal{P} ; s)$ is close to the expected number $|\mathcal{P}|^{3} / q$.
\end{abstract}

\section{Introduction}

Let $\mathcal{P}$ be a set of $n$ points in the plane $\mathbb{R}^{2}$. We consider all the triangles whose vertices are any three non-collinear points of $\mathcal{P}$. We regard these triangles as triangles determined by the set $\mathcal{P}$. Denote by $g(\mathcal{P})$ the number of distinct areas of triangles determined by $\mathcal{P}$. For every $n \in \mathbb{N}$, let $g(n)$ be the minimum of $g(\mathcal{P})$ over all sets $\mathcal{P}$ of $n$ noncollinear points in the plane. The first estimates on $g(n)$ were given by Erdős and Purdy [7], who proved that

$$
c_{1} n^{3 / 4} \leq g(n) \leq c_{2} n
$$

for some absolute constant $c_{1}, c_{2}>0$. The upper bound ([1]) can be archived by taking $\mathcal{P}$ to be a set of equally space $\left\lceil\frac{n}{2}\right\rceil$ points on a line $l$ together with $\left\lfloor\frac{n}{2}\right\rfloor$ equally spaced points on a line $l^{\prime}$ parallel to $l$. It gives $\left\lfloor\frac{n-1}{2}\right\rfloor$ triangles of distinct areas. Motivated by this example, Erdős, Purdy, and Straus [8] conjectured the following:

Conjecture 1.1 ([8]) For every $n, g(n)=\left\lfloor\frac{n-1}{2}\right\rfloor$.

A linear lower bound was first established by Burton and Purdy [1]. More precisely, they showed that $g(n) \geq 0.32 n$. Dumitrescu and Cs. Tóth [5] improved this bound to 
$g(n) \geq \frac{17}{38} n-O(1) \approx 0.04473$. Pinchasi [18] settled Conjecture 1.1 using an ingenious counting argument. Recently, a stronger version of the result of Pinchasi is proved by Iosevich and Rudnev [13].

The remarkable results of Bourgain, Katz and Tao [2] on sum-product problem and its application in Erdős distance problem over finite fields have stimulated a series of studies of finite field analogues of classical discrete geometry problems, see [3, 4, 6, 9, 11, 12, 14 , $15,16,13,19,20,21,22,23,24]$ and references therein. The main purpose of this note is to study the finite field analogue of $g(n)$. Since there are only $q$ possible areas for triangles in finite plane $\mathbb{F}_{q}^{2}$, one may expect that if $\mathcal{P}$ is large, then $\mathcal{A}(\mathcal{P})$ covers the whole or a positive proportion of $\mathbb{F}_{q}$.

Let $\mathcal{P}$ be a set of $n$ points in the finite plane $\mathbb{F}_{q}^{2}$ over the finite field $\mathbb{F}_{q}$ of $q$ elements, where $q$ is an odd prime power. The area of triangles determined by three points $\boldsymbol{x}=$ $\left(x_{1}, x_{2}\right), \boldsymbol{y}=\left(y_{1}, y_{2}\right), \boldsymbol{z}=\left(z_{1}, z_{2}\right) \in \mathbb{F}_{q}^{2}$ is defined as usual

$$
\frac{1}{2}\left|\begin{array}{lll}
x_{1} & x_{2} & 1 \\
y_{1} & y_{2} & 1 \\
z_{1} & z_{2} & 1
\end{array}\right|=\frac{1}{2}(\boldsymbol{x} * \boldsymbol{y}+\boldsymbol{y} * \boldsymbol{z}+\boldsymbol{z} * x),
$$

where $\left(a_{1}, a_{2}\right) *\left(b_{1}, b_{2}\right)=a_{1} b_{2}-a_{2} b_{1} \in \mathbb{F}_{q}$. For any $s \in \mathbb{F}_{q}$, denote by $A(\mathcal{P} ; s)$ the number of ordered triangles (i.e. triangles are determined by an ordered triple of vertices) whose vertices in $\mathcal{P}$ having area $s$. Our main result is the following theorem.

Theorem 1.2 Let $\mathcal{P}$ be a set of $n$ points in the finite plane $\mathbb{F}_{q}^{2}$. Suppose that $s \neq 0$ and $|\mathcal{P}| \gg q^{3 / 2}$ then $A(\mathcal{P} ; s)=(1+o(1))|\mathcal{P}|^{3} / q$. Moreover, if $|\mathcal{P}| \gg q^{5 / 3}$ then $A(\mathcal{P} ; 0)=$ $(1+o(1))|\mathcal{P}|^{3} / q$.

We conjecture that the exponent $3 / 2$ can be further improved to $1+\epsilon$, or at least, we hope to show that if $|\mathcal{P}| \gg q^{1+\epsilon}$ then we can find a triangle of an arbitrary area from the set $\mathcal{A}$. Note that one can not go lower than 1 as we can take $q$ points on a line.

In the nondegenerate case $s \neq 0$, the above result follows immediately from Hart and Iosevich's result ([9]) on the problem where one of the vertices of the triangle is assumed to be at the origin. Our result in the degenerate case $s=0$, however, is stronger than the related result in Hart and Iosevich ([9]).

\section{Proof of Theorem 1.2}

To prove the first part of Theorem 1.2 we will need the following lemma.

Lemma 2.1 Let $\mathcal{P}$ be a set of $n$ points in the finite plane. For any $s \in \mathbb{F}_{q}$, denote by $\mu(\mathcal{P}, s)$ the number of ordered pair $(\boldsymbol{x}, \boldsymbol{y}) \in \mathcal{P} \times \mathcal{P}$ such that $\boldsymbol{x} * \boldsymbol{y}=2$ s. For any $s \neq 0$, then

$$
\left|\mu(\mathcal{P}, s)-\frac{|\mathcal{P}|^{2}}{q}\right| \leq 2 \sqrt{q}|\mathcal{P}| .
$$


Proof See the proof of Theorem 1.4 in [10]. Note that [10, Theorem 1.4] only states for dot product but the proof works transparently for any non-degenerate bilinear form, in particular, the $*$-product defined here. The reader also can find a graph-theoretic proof of this lemma in [24, Section 8].

For any $\boldsymbol{x} \in \mathcal{P}$, denote $\mathcal{P}-\boldsymbol{x}=\{\boldsymbol{y}-\boldsymbol{x}: \boldsymbol{y} \in \mathcal{P}\}$. Then

$$
A(\mathcal{P} ; s)=\sum_{\boldsymbol{x} \in \mathcal{P}} \mu(\mathcal{P}-\boldsymbol{x}, s)
$$

It follows from Lemma 2.1 that $\mu(\mathcal{P}-\boldsymbol{x}, s)=(1+o(1)) \frac{|\mathcal{P}|^{2}}{q}$ if $|\mathcal{P}| \gg q^{3 / 2}$. Together with $(2.1)$, we have $A(\mathcal{P} ; s)=(1+o(1))|\mathcal{P}|^{3} / q$ if $|\mathcal{P}| \gg q^{3 / 2}$.

Next we will follow the methods in [19] to prove the second part of the theorem. Note that Lemma 2.1 does not work when $s=0$, so we cannot use the above methods to study the case of triangles with zero area. Let $\Psi$ be the set of all additive characters of $\mathbb{F}_{q}$, and let $\Psi^{*} \subset \Psi$ be the set of all nonprincipal characters. We recall the following identity

$$
\sum_{\psi \in \Psi} \psi(z)= \begin{cases}q & z=0 \\ 0 & \text { otherwise }\end{cases}
$$

Note that if the field is $\mathbb{Z}_{p}$, then the characters are just $e^{\frac{2 \pi i a}{p}}$ and the identity follows by summing up the geometric series. For more information about the additive characters, we refer the reader to [17, Section 11.1].

For any $\boldsymbol{a}, \boldsymbol{b}, \boldsymbol{c} \in \mathbb{F}_{q}^{2}$ and $\lambda \in \mathbb{F}_{q}$, the product $*$ satisfies the following properties:

$$
\begin{aligned}
\boldsymbol{a} * \boldsymbol{b} & =-\boldsymbol{b} * \boldsymbol{a} \\
\boldsymbol{a} * \boldsymbol{b}+\boldsymbol{a} * \boldsymbol{c} & =\boldsymbol{a} *(\boldsymbol{b}+\boldsymbol{c}) \\
\boldsymbol{a} *(\lambda \boldsymbol{b}) & =\lambda(\boldsymbol{a} * \boldsymbol{b}) .
\end{aligned}
$$

It follows from (1.2) and (2.2) that

$$
A(\mathcal{P} ; s)=\frac{1}{q} \sum_{\psi \in \Psi} \sum_{\boldsymbol{x}, \boldsymbol{y}, \boldsymbol{z} \in \mathcal{P}} \psi(\boldsymbol{x} * \boldsymbol{y}+\boldsymbol{y} * \boldsymbol{z}+\boldsymbol{z} * \boldsymbol{x}-2 s) .
$$

Separating the principle character gives

$$
\begin{aligned}
\left|A(\mathcal{P} ; 0)-\frac{|\mathcal{P}|^{3}}{q}\right| & =\frac{1}{q}\left|\sum_{\psi \in \Psi^{*}} \sum_{\boldsymbol{x}, \boldsymbol{y}, \boldsymbol{z} \in \mathcal{P}} \psi(\boldsymbol{x} * \boldsymbol{y}+\boldsymbol{y} * \boldsymbol{z}+\boldsymbol{z} * \boldsymbol{x})\right| \\
& \leq \frac{1}{q} \sum_{\psi \in \Psi^{*}}\left|\sum_{\boldsymbol{x}, \boldsymbol{y}, \boldsymbol{z} \in \mathcal{P}} \psi(\boldsymbol{x} *(\boldsymbol{y}-\boldsymbol{z})+\boldsymbol{y} * \boldsymbol{z})\right|
\end{aligned}
$$


Applying the Cauchy-Schwartz inequality twice, we have

$$
\begin{aligned}
& \leq\left|A(\mathcal{P} ; 0)-\frac{|\mathcal{P}|^{3}}{q}\right|^{2} \\
& \leq \frac{q-1}{q^{2}} \sum_{\psi \in \Psi^{*}}\left|\sum_{\boldsymbol{x}, \boldsymbol{y}, \boldsymbol{z} \in \mathcal{P}} \psi(\boldsymbol{x} *(\boldsymbol{y}-\boldsymbol{z})+\boldsymbol{y} * \boldsymbol{z})\right|^{2} \\
& <\frac{(q-1)|\mathcal{P}|}{q^{2}} \sum_{\psi \in \Psi^{*}} \sum_{\boldsymbol{x} \in \mathcal{P}}\left|\sum_{\boldsymbol{y}, \boldsymbol{z} \in \mathcal{P}} \psi(\boldsymbol{x} *(\boldsymbol{y}-\boldsymbol{z})+\boldsymbol{y} * \boldsymbol{z})\right|^{2} \\
& \leq \frac{(q-1)|\mathcal{P}|}{q^{2}} \sum_{\psi \in \Psi^{*}} \sum_{\boldsymbol{x} \in \mathbb{F}_{q}^{2}}\left|\sum_{\boldsymbol{y}, \boldsymbol{z} \in \mathcal{P}} \psi(\boldsymbol{x} *(\boldsymbol{y}-\boldsymbol{z})+\boldsymbol{y} * \boldsymbol{z})\right|^{2} \\
& =\frac{(q-1)|\mathcal{P}|}{q^{2}} \sum_{\psi \in \Psi^{*}} \sum_{\boldsymbol{x} \in \mathbb{F}_{q}^{2}} \sum_{\boldsymbol{y}, \boldsymbol{z}, \boldsymbol{y}^{\prime}, \boldsymbol{z}^{\prime} \in \mathcal{P}} \psi\left(\boldsymbol{x} *\left(\boldsymbol{y}-\boldsymbol{z}-\boldsymbol{y}^{\prime}+\boldsymbol{z}^{\prime}\right)+\boldsymbol{y} * \boldsymbol{z}-\boldsymbol{y}^{\prime} * \boldsymbol{z}^{\prime}\right) \\
& =\frac{(q-1)|\mathcal{P}|}{q^{2}} \sum_{\psi \in \Psi^{*}} \sum_{\boldsymbol{y}, \boldsymbol{z}, \boldsymbol{y}^{\prime}, \boldsymbol{z}^{\prime} \in \mathcal{P}} \psi\left(\boldsymbol{y} * \boldsymbol{z}-\boldsymbol{y}^{\prime} * \boldsymbol{z}^{\prime}\right) \sum_{\boldsymbol{x} \in \mathbb{F}_{q}^{2}} \psi\left(\boldsymbol{x} *\left(\boldsymbol{y}-\boldsymbol{z}-\boldsymbol{y}^{\prime}+\boldsymbol{z}^{\prime}\right)\right) .
\end{aligned}
$$

By the orthogonality property of additive characters (2.2), we see that the inner sum vanishes if and only if $\boldsymbol{y}-\boldsymbol{z}=\boldsymbol{y}^{\prime}-\boldsymbol{z}^{\prime}$, in which case it equals $q^{2}$. This implies that

$$
\begin{aligned}
\left|A(\mathcal{P} ; 0)-\frac{|\mathcal{P}|^{3}}{q}\right|^{2} & \leq(q-1)|\mathcal{P}| \sum_{\psi \in \Psi^{*}} \sum_{\boldsymbol{y}, \boldsymbol{z}, \boldsymbol{y}^{\prime}, \boldsymbol{z}^{\prime} \in \mathcal{P}, \boldsymbol{y}-\boldsymbol{z}=\boldsymbol{y}^{\prime}-\boldsymbol{z}^{\prime}} \psi\left(\boldsymbol{y} * \boldsymbol{z}-\boldsymbol{y}^{\prime} * \boldsymbol{z}^{\prime}\right) \\
& \leq(q-1)|\mathcal{P}| \sum_{\psi \in \Psi} \sum_{\boldsymbol{y}, \boldsymbol{z}, \boldsymbol{y}^{\prime}, \boldsymbol{z}^{\prime} \in \mathcal{P}, \boldsymbol{y}-\boldsymbol{z}=\boldsymbol{y}^{\prime}-\boldsymbol{z}^{\prime}} \psi\left(\boldsymbol{y} * \boldsymbol{z}-\boldsymbol{y}^{\prime} * \boldsymbol{z}^{\prime}\right) \\
& =q(q-1)|\mathcal{P}| V,
\end{aligned}
$$

where $V$ is the number of solutions to the system

$$
\boldsymbol{y}-\boldsymbol{z}=\boldsymbol{y}^{\prime}-\boldsymbol{z}^{\prime} \text { and } \boldsymbol{y} * \boldsymbol{z}=\boldsymbol{y}^{\prime} * \boldsymbol{z}^{\prime}
$$

in $\boldsymbol{y}, \boldsymbol{z}, \boldsymbol{y}^{\prime}, \boldsymbol{z}^{\prime} \in \mathcal{P}$. We consider two cases.

Case I. Suppose that $\boldsymbol{y}=\boldsymbol{z}$ or $\boldsymbol{y}^{\prime}=\boldsymbol{z}^{\prime}$. Since $\boldsymbol{y}=\boldsymbol{z}$ if and only if $\boldsymbol{y}^{\prime}=\boldsymbol{z}^{\prime}$, this case contributes $|\mathcal{P}|^{2}$ solutions to the system (2.4).

Case II. Suppose that $\boldsymbol{y} \neq \boldsymbol{z}$ and $\boldsymbol{y}^{\prime} \neq \boldsymbol{z}^{\prime}$. The number of solutions to the system (2.4) in this case is bounded by the number of solutions to the equation

$$
\boldsymbol{y} * \boldsymbol{z}=\boldsymbol{y}^{\prime} *\left(\boldsymbol{y}^{\prime}+\boldsymbol{z}-\boldsymbol{y}\right)=\boldsymbol{y}^{\prime} *(\boldsymbol{z}-\boldsymbol{y})
$$

in $\boldsymbol{y}, \boldsymbol{z}, \boldsymbol{y}^{\prime} \in \mathcal{P}, \boldsymbol{y} \neq \boldsymbol{z}$. When $\boldsymbol{y}, \boldsymbol{z} \in \mathcal{P}, \boldsymbol{y} \neq \boldsymbol{z}$ are fixed, the equation (2.5) has at most $q$ solutions. Hence, this case contributes at most $q|\mathcal{P}|^{2}$ solutions to the system (2.4).

Putting these cases together, we have

$$
W \leq(q+1)|\mathcal{P}|^{3} .
$$


It follows from (2.3) and (2.6) that

$$
\left|A(\mathcal{P} ; 0)-\frac{|\mathcal{P}|^{3}}{q}\right|^{2} \leq q(q-1)(q+1)|\mathcal{P}|^{3}<q^{3}|\mathcal{P}|^{3},
$$

which also implies that $A(\mathcal{P} ; 0)=(1+o(1)) \frac{|\mathcal{P}|^{3}}{q}$ if $|\mathcal{P}| \gg q^{5 / 3}$. This completes the proof of Theorem 1.2 .

\section{References}

[1] G. R. Burton and G. B. Purdy, The directions determined by $n$ points in the plane, J. London Math. Soc. 20 (1979), 109-114.

[2] J. Bourgain, N. Katz, and T. Tao, A sum product estimate in finite fields and Applications, Geom. Funct. Analysis, 14 (2004), 27-57.

[3] J. Chapman, M. B. Erdogan, Derrick Hart, Alex Iosevich, and Doowon Koh, Pinned distance sets, $k$-simplices, Wolff's exponent in finite fields and sum-product estimates, Mathematische Zeitschrift (to appear).

[4] D. Covert, D. Hart, A. Iosevich and I. Uriarte-Tuero, An analog of the FurstenbergKatznelson-Weiss theorem on triangles in sets of positive density in finite field geometries, European Journal of Combinatorics, to appear.

[5] A. Dumitrescu and Cs. D. Tóth, Distinct triangles areas in a planar point set, Disc. and Comp. Geom., to appear.

[6] Z. Dvir, On the size of Kakeya sets in finite fields, J. Amer. Math. Soc., 22 (2009), 1093-1097.

[7] P. Erdős and G. Purdy, Some extremal problems in geometry V., Proceedings of the Eighth Southeastern Conference on Combinatorics, Graph Theory and Computing (Louisiana State Univ., Baton Rouge, La., 1979), 569-578.

[8] P. Erdős, G. Purdy, and E. G. Strauss, On a problem in combinatorial geometry, Discrete Mathematics 40 (1982), 45-52.

[9] D. Hart and A. Iosevich, Ubiquity of simplices in subsets of vector spaces over finite fields, Analysis Mathematika, 34 (2007).

[10] D. Hart and A. Iosevich, Sums and products in finite fields: an integral geometric viewpoint, Contemporary Mathematics: Radon transforms, geometry, and wavelets, 464, RI (2008).

[11] D. Hart, A. Iosevich, D. Koh and M. Rudnev, Averages over hyperplanes, sumproduct theory in vector spaces over finite fields and the Erdős-Falconer distance conjecture, Trans. Amer. Math. Soc, 363 (2011), 3255-3275.

[12] A. Iosevich and D. Koh, Erdős-Falconer distance problem, exponential sums, and Fourier analytic approach to incidence theorem in vector spaces over finite fields, SIAM J. Disc. Math., 23 (2008), 123-135. 
[13] A. Iosevich, O. Roche-Newton, M. Rudnev, On an application of Guth-Katz theorem, Math. Res. Lett, 18 (2011), no. 4, pp 1-7.

[14] A. Iosevich and M. Rudnev, Erdős distance problem in vector spaces over finite fields, Trans. Amer. Math. Soc., 359 (2007), 6127-6142.

[15] A. Iosevich, I. E. Shparlinski, and M. Xiong, Sets with integral distances in finite fields, Trans. Amer. Math. Soc. 362 (2010), 2189-2204.

[16] A. Iosevich and S. Senger, Orthogonal systems in vector spaces over finite fields, The Electronic J. Combin., 15 (2008), Article R151.

[17] H. Iwaniec and E. Kowalski, Analytic number theory, Amer. Math. Soc., Providence, RI, 2004.

[18] R. Pinchasi, The minimum number of distinct areas of triangles determined by a set of $n$ points in the plane, SIAM J. Disc. Math., 22 (2008), 828-831.

[19] I. E. Shparlinski, On Point Sets in Vector Spaces over Finite Fields that Determine only Acute Angle, Bull. Aust. Math. Soc., 81 (2010), 114-120.

[20] L. A. Vinh, Explicit Ramsey graphs and Erdős distance problem over finite Euclidean and non-Euclidean spaces, Electronic J. Combin., 15 (2008), Aricle R5.

[21] L. A. Vinh, On the number of orthogonal systems in vector spaces over finite fields, Electronic J. Combin., 15 (2008), Aricle N32.

[22] L. A. Vinh, On a Furstenberg-Katznelson-Weiss type theorem over finite fields, Annals of Combinatorics, 15 (2011), 541-547,

[23] L. A. Vinh, On $k$-simplexes in $(2 k-1)$-dimensional vector spaces over finite fields, Discrete Mathematics and Theoretical Computer Science Proc. AK, 2009, 871-880.

[24] L. A. Vinh, The sovability of norm, bilinear and quadratic equations over finite fields via spectra of graphs, arXiv:0904.0441. 